\title{
Aspectos semióticos inherentes a la indización de los recursos de información
}

\author{
Inherent semiotic aspects to the indexation of information resources
}

\begin{abstract}
Adriana SUÁREZ SÁNCHEZ
Instituto de Investigaciones Bibliotecológicas y de la Información, Universidad Nacional Autónoma de México. Circuito Interior de Ciudad Universitaria s/n, piso 12 de la Torre II de Humanidades, C.P. 04510, Alcaldía de Coyoacán, Ciudad de México, México, asuarez@iibi.unam.mx
\end{abstract}

\begin{abstract}
Resumen
El objetivo del artículo es identificar los elementos semióticos que se encuentran presentes en la indización de los recursos de información. La metodología empleada consistió en el análisis del modelo semiótico de Charles Sanders Peirce y su comparación con el proceso de indización de los recursos de información, según la teoría planteada por Isidoro Gil Leiva. Se encontró que en la indización de los recursos de información subyacen los siguientes elementos semióticos: un Objeto (lo que es representado), un representador (quien representa), un Signo (la representación) y un Interpretante (lo que el Signo crea en alguien a quien se dirige). La representación temática de los recursos de información incluye elementos semióticos fundamentales a la vez que se caracteriza por un Objeto abstracto e imbuido en un recurso de información, un representador con perfil profesionalizante y un Signo, limitado por el frecuente uso de lenguajes artificiales para la simbolización del Objeto. Se concluye que el estudio de la semiótica es importante para los bibliotecólogos, en vista de que la teoría del Signo guarda relación con la representación y comunicación de entidades de información. A partir de la función representadora, el Signo se concreta en registros bibliográficos que refieren a los recursos en un sistema. Derivado de la función comunicativa, el Signo es un puente entre las necesidades de información de los usuarios y los recursos.
\end{abstract}

Palabras clave: Semiótica. Indización. Representación temática. Peirce, Charles Sanders.

\section{Introducción}

La semiótica ha sido definida como la "ciencia que estudia las diferentes clases de signos, así como las reglas que gobiernan su generación y producción, transmisión e intercambio, recepción e interpretación" (Serrano, 1981, p. 7).

Originalmente el término estuvo asociado con la medicina y la interpretación de los signos que los primeros médicos de occidente registraban para comprender la interacción entre aspectos fisiológicos y mentales (Ferrater Mora, 1999). Durante la Edad Media y el Renacimiento, los estudios del Signo fueron importantes y destacaron los trabajos de San Agustín quien lo

\begin{abstract}
The purpose of this paper is to identify the semiotic elements which conform the theoretical basis for indexing information resources. The methodology used in this research was based in the semiotic model of Charles Sanders Peirce and his comparison with the indexation process according with the theory posed by Isidoro Gil Leiva. It was found that the indexation of information resources underlies the following semiotic elements: an Object (what is represented), a representative (he who represents), a Sign (the representation) and an interpreter (what the sign creates in someone to whom it directs). The thematic representation of resources includes the fundamental semiotic elements at the same time which is characterized by an abstract Object and is imbued in a bibliographical support, a representative with a professionalizing profile and a Sign, limited by the frequent use of artificial languages for symbolizing the Object. It is concluded that the study of semiotics is important for librarians, in view that the theory of sign refers to the representation and communication of information entities. Departing from the representing function, the Sign materializes in bibliographic records which substitute the resources in a system. Emanating from the communicative function, the sign is a bridge between the needs of information of users and the resources.
\end{abstract}

Keywords: Semiotics. Indexing. Subject representation. Peirce, Charles Sanders.

asumió como un elemento representador de la realidad, Leibnitz que estudió el Signo en lenguas especializadas: matemáticas y lenguaje de sordomudos y Thomas Hobbes que estudió la semiótica en relación con el orden de la representación y el conocimiento humano.

La época moderna fue especialmente importante para la semiótica debido al giro que experimentaron los estudios lingüísticos a partir de los postulados de la lingüística sincrónica, el estructuralismo y el pragmatismo lingüístico (Escavy Zamora, 2018). Entre 1850 y 1920 la teoría del Signo se nutrió de las teorías de dos 
autores: Ferdinand de Sauusure y Charles Sanders Peirce.

Ferdinand de Sauusure (1857-1913) en su obra Curso de lingüística general (1961, p. 43) se adelanta al surgimiento de la semiótica cuando menciona que:

Se puede, pues, concebir una ciencia que estudie la vida de los signos en el seno de la vida social. Tal ciencia sería parte de la psicología social, y por consiguiente de la psicología general. Nosotros la llamaremos semiología (del griego sēmeîon 'signo'). Ella nos enseñará en qué consisten los signos y cuáles son las leyes que los gobiernan. Puesto que todavía no existe, no se puede decir qué es lo que ella será; pero tiene derecho a la existencia, y su lugar está determinado de antemano.

Charles Sanders Peirce (1974, parágrafo 2.228) concibió la semiótica como la ciencia general que estudia el signo y estableció que: "Un signo, o representamen, es algo que está para alguien, por algo, en algún aspecto o disposición."

A diferencia de Saussure que vinculó directamente la semiótica con el signo lingüístico, Peirce lo inscribe en un contexto más amplio que será fundamental para su inserción en áreas de conocimiento diversas.

Desde una perspectiva reducida, la semiótica estudia el Signo y los sistemas de signos (Feather \& Sturges, 2003; Martínez de Souza, 2004; Thiebau, 2007). Desde un enfoque más amplio, analiza todas aquellas representaciones simbólicas asociadas con procesos de representación y comunicación (Ogden \& Richards, 1953; Peirce, 1974). Tenemos entonces que (Sebeok, 1996, p. 12):

La semiótica es el término comúnmente utilizado para referirnos al estudio de la capacidad innata de los seres humanos para producir y comprender signos de todas clases (desde los que pertenecen a simples sistemas de signos psicológicos hasta aquellos que revelan una estructura simbólica altamente compleja.

En este sentido (Toledo Almada y Sequera Meza, 2014, p. 1):

[...] puede explicar tanto los fenómenos de la vida social ordinaria como los procesos por los cuales científicos, artistas, teólogos, brujos y chamanes, el hombre cotidiano, construyen cuerpos de conocimiento tendientes a dar al ser humano explicaciones sobre la existencia, naturaleza de las cosas del mundo (tanto las tangibles como las intangibles); es decir, toda la vida del hombre en tanto cadena productora de una realidad social construida a través del lenguaje.

Todo proceso semiótico posee rasgos epistemológicos y comunicativos. A partir de sus rasgos epistemológicos, el hombre construye representaciones asibles (concretadas en signos) de la realidad abrumadora e inabarcable. Tras la acción, se descubre una naturaleza humana que, al no poder aprehender los objetos tal como existen en la realidad, trata de reducir lo complejo a simple y lo confuso a unidades claras y comprensibles. A partir de sus rasgos comunicativos, el proceso semiótico reduce entidades complejas en signos capaces de transmitir información necesaria para la construcción de ideas, pensamientos, relaciones, etc., creando un entramado social. Desde Aristóteles se tiene la costumbre de decir que el hombre es un animal social; en este marco, el Signo es parte del circuito y se torna una herramienta que los interlocutores emplean para transmitir su pensamiento.

Originalmente, la semiótica estuvo vinculada con la filosofía, sin embargo, al paso del tiempo se convirtió en un campo transdisciplinar. Actualmente, muchas áreas de conocimiento se han acercado a las bases semióticas para comprender la función de los signos (icónicos, lingüísticos, numéricos, etc.) en su contexto de aplicación. Tal es el caso de la comunicación, la psicología, la política, la literatura, los estudios de mercado, etc.

En comparación con otras disciplinas, la bibliotecología se ha interesado poco en la semiótica. En área es posible mencionar: (1) la corriente de semántica documental iniciada a principios de los noventas por Izquierdo Arroyo (1993) que contempló el modelo semiótico de Peirce aplicado a las ciencias del texto, (2) los elementos semióticos de la representación del conocimiento establecidos por Birger Hjørland en Information Seeking and Subject Representation (1997), (3) los principios epistémicos, semióticos y lingüísticos relacionados con la organización de recursos de información establecidos por Elaine Svenonius en The Intelectual Foundations of Information Organization (2000), (4) los estudios sobre indización y semiótica de Jens-Erik Mai a principios de milenio (Mai, 2000; Mai, 2001) y (5) recientemente los trabajos de Almeida, Lopes Fujita y Dos Reis (2013) sobre el pensamiento de Peirce y la indización.

No obstante, hace falta mayor investigación sobre el tema en vista de que los bibliotecólogos somos representadores de recursos de información, lo que implica que utilizamos signos como sustitutos de "algo" y los dirigimos a "alguien" con fines comunicativos. Es importante considerar que si bien "los estudios sobre métodos para organizar la información y el conocimiento algunas veces menosprecian las interconexiones teóricas entre las ciencias de la información y otras disci- 
plinas" (Almeida, 2012, p. 4), muchos fundamentos teóricos de otros campos pueden enriquecer la teoría de las ciencias documentales.

Dicho esto, el interés de la bibliotecología en la semiótica reside en, al menos, dos aspectos: 1) que la disciplina bibliotecaria se vale de la representación mediante signos como herramienta para simbolizar entidades de información en sistemas; y 2) que las representaciones creadas mediante signos constituyen puentes comunicativos con los usuarios de la biblioteca.

Considerando lo anterior, el presente estudio tiene por objetivo identificar los elementos semióticos presentes en la indización de los recursos de información a partir del análisis contrastivo de un modelo de representación semiótica (modelo semiótico de Peirce) y el proceso de indización (teoría de indización de Gil Leiva).

El supuesto del que se partió fue que la indización al ser una representación de algo incluye elementos semióticos esenciales, esto es, un Objeto, un Signo, un Interpretante, pero posee elementos particulares del dominio bibliotecológico que los caracterizan y/o reconceptualizan.

\section{Metodología}

La metodología empleada para identificar los aspectos semióticos intrínsecos en la indización de los recursos de información se formó de dos técnicas:

1. Método analítico: es una forma de investigación que implica habilidades de pensamiento crítico y la evaluación de hechos e información. Se enfoca en la identificación de los elementos principales detrás del tema que se está analizando para comprenderlo adecuadamente.

2. Análisis contrastivo: es el método comparativo de dos estructuras de investigación. Una puede ser analizada a la luz de otra o bien se comparan elementos previamente establecidos.

El estudio se constituyó de tres etapas:

1. Análisis del modelo semiótico planteado por Charles Sanders Peirce en su obra La ciencia de la semiótica (1974). En la selección del modelo semiótico que se emplearía se revisaron diversas propuestas: Ogden y Richards, Morris y Ullman. Entre ellas, se optó por el modelo de Peirce porque se adhiere a la función pragmática del signo en tanto que asocia el signo con un "alguien" que recupera el signo y le confiere un significado. Por demás, como señala Almeida (2012), la propuesta de Peirce posibilita establecer vínculos entre la ciencia de la información y la semiótica porque contiene elementos claves que las ciencias documentales contemplan en la organización y recuperación temática de los recursos de información. Se recuperaron sus elementos constitutivos, las definiciones asociadas a éstos y el flujo del proceso semiótico.

2. Análisis del proceso de representación temática de recursos de información, con base en la teoría establecida por Isidoro Gil Leiva en Manual de indización: teoría y práctica (2008). Se seleccionó la teoría establecida por Gil Leiva debido a su amplia difusión y aplicación en el contexto hispanoamericano. Por demás, se optó por esta teoría indizadora porque es un modelo generalista que en comparación con otras teorías no incluye deliberadamente aspectos semióticos. Se determinaron sus elementos constitutivos, los aspectos que los caracterizan y el flujo del proceso.

El alcance de esta investigación se limitó al análisis de un modelo de indización general; sin embargo, tras los resultados, se vislumbra como una investigación futura y necesaria la comparación de los aspectos semióticos subyacentes en modelos generales de indización y modelos de indización que consideren intencionalmente aspectos semióticos (Almeida, Mai, etc.).

3. Análisis contrastivo entre el modelo semiótico y el proceso de indización para: 1) determinar los elementos semióticos que subyacen en la indización y 2) observar el desarrollo del proceso con la intención de establecer semejanzas y detectar diferencias.

\section{Resultados}

Después del análisis, constituido por tres etapas: (1) el análisis del modelo semiótico planteado por Charles Sanders Peirce, (2) el escrutinio del proceso de representación temática de recursos de información, según la teoría de Gil Leiva (2008) y (3) el análisis contrastivo entre el modelo semiótico y el proceso de indización, se obtuvieron un conjunto de resultados que se exponen en los apartados siguientes.

En el primero, se analiza el modelo semiótico de Peirce a partir de sus elementos constitutivos y como proceso. En el segundo, se examinan los elementos de la representación temática de recursos de información y el desarrollo de la actividad. Por último, se comparan ambos y se identifican los elementos semióticos que subyacen en la indización de los recursos de información.

\subsection{El modelo semiótico de Charles S. Peirce}

Charles Sanders Peirce (1839-1914) fue un filósofo, lógico y científico estadounidense fundador del pragmatismo filosófico. Su trabajo constituye 
una piedra angular en el desarrollo de la semiótica en vista de que (Conesa \& Nubiola, 2002, p. 52):

Muchos de los temas y avances que han emergido en la filosofía de la ciencia, del lenguaje y de la acción más recientes no sólo fueron anticipados por Peirce, sino también en muchos casos explorados por él con rigor y profundidad [...] De hecho, la mejor manera de entender a Peirce es considerar que aspiraba a hacer algo similar a la filosofía contemporánea, anticipando el giro lingüístico de la filosofía mediante su teoría general de los signos.

Antes de Peirce, Saussure había establecido los elementos significante-significado como las dos caras de la moneda de los signos; sin embargo, su proposición dejó fuera un elemento clave: el Interpretante. Peirce rechazó tajantemente el dualismo cartesiano y la noción de que todo pensamiento era percepción interna de ideas (Conesa \& Nubiola, 2002) y formuló un proceso semiótico fundamentado en la triada Objeto-SignoInterpretante.

Según Peirce la noción de Signo no se fundamenta solamente en la equivalencia entre significante ("casa"- la palabra escrita) y significado (edificación destinada para ser habitada), entre expresión y contenido, sino en la inferencia, la interpretación, la dinámica de la semiosis que es "un proceso en que, en ejercicio de la facultad semiótica, el sujeto da forma a los símbolos y signos, y los produce con su consiguiente significación" (Niño Rojas, 2013, p. 286).

La semiosis considera la producción de sentido a partir del Signo, es decir, transitar más allá del significante al significado, buscar y producir, interpretar el mundo y comunicarlo. En la construcción planteada por Peirce opera un proceso de semiosis en el que (Vidales Gonzáles, 2010, p. 93):

[...] un signo o representamen es aquello que está Primero en relación con un Segundo, su objeto, el cual tiene la capacidad de determinar un Tercero, el interpretante, que de hecho asume la misma relación triádica con el objeto. Peirce entiende a la semiosis como la acción o influencia que envuelve una cooperación entre tres elementos: un signo, su objeto y su interpretante, la cual no puede ser resuelta en acciones entre pares.

Peirce planteó que el proceso semiótico es inherente a la habilidad de la mente para transformar las impresiones de los sentidos en construcciones significativas particulares. Desde su aparición, su modelo ha sido altamente efectivo para explicar el modo como son producidos e interpretados los signos (Sebeok, 1996) y contempla los siguientes elementos constitutivos (Figura 1):

a) Objeto: es aquello acerca de lo cual el Signo presupone un conocimiento para que sea posible proveer alguna información adicional sobre el mismo. Los objetos pueden ser una cosa singular conocida existente, que se cree que haya existido, que se espera que exista, hechos conocidos, una cualidad o relación de hechos conocidos o un conjunto de tales cosas.

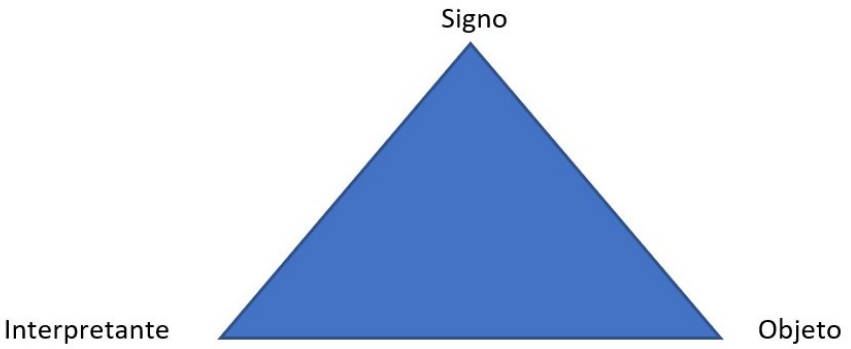

Figura 1. Elementos constitutivos del modelo semiótico de Peirce

b) Signo: es algo que, para alguien, representa o se refiere a algo en algún aspecto o carácter. Está en lugar de algo, su Objeto.

c) Interpretante: es la determinación del Signo realizada por alguien o algo a quien el Signo es dirigido.

El Signo está "en alguna relación con algo -su Objeto" y "para alguien -su Interpretante". De modo que, "la oración "Caín mató a Abel", que es un Signo, se refiere tanto a Caín como a Abel [como objetos], aún si no se considera -como debería- que se tiene un matar como tercer Objeto" (Peirce, 1974, p. 23). Y tal Signo pueden tener interpretantes diversos a partir de la idea que generan en ese "alguien" a quien se dirigen. En este caso, la determinación del Signo bien puede ser que alguien llamado Abel dio muerte, quitó la esencia de vida, a alguien Ilamado Caín o bien como una sentencia asociada con la traición entre dos sujetos con lazos cercanos.

De manera más esquematizada y sencilla, si tenemos el Signo "Árbol" detrás pueden subyacen los siguientes aspectos en calidad de Objeto e Interpretante (Figura 2).

El modelo semiótico de Peirce es una teoría del Signo a la vez que una teoría representativa-comunicacional sustentada en tres elementos: un Objeto que puede estar asociado a lo real, abstracto, pensamiento, etc.; un Signo, establecido bajo cierto código (que puede ser lingüístico, numérico, icónico, pictórico, etc.) y un Interpretante, determinado por "a quien el Signo se dirige" y en quien crea una idea en la que confluyen elementos cognitivos, sociales, políticos, psicológicos, culturales, etc. 


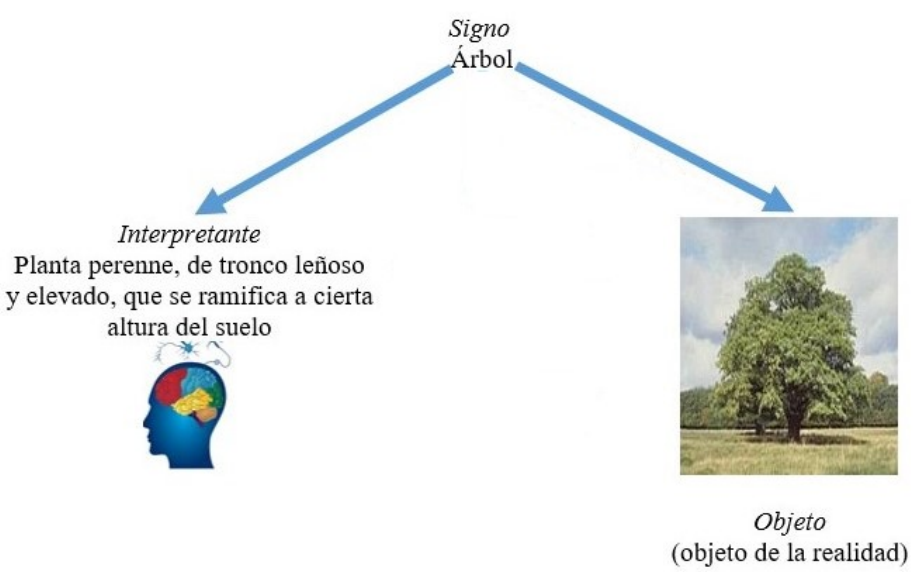

Figura 2. Aplicación del modelo semiótico de Peirce

Los signos, según el modelo de Peirce, se agrupan en nueve clases:

i) Cualisigno: se trata de un signo que toma del objeto y transfiere a un interpretante el mero aspecto formal de tal objeto.

ii) Ícono: es un signo que se refiere al Objeto que denota tan solo en virtud de los caracteres que le son propios, y que éste posee por igual con independencia de la existencia o no existencia actual de cada objeto.

iii) Rhema: es un signo que, para su interpretante, es Signo de posibilidad cualitativa, es decir, que se lo entiende como la representación de tal o cual tipo de Objeto posible.

iv) Sinsigno: es una cosa o existencia de acontecimiento actual, la cual es un signo.

v) Índice: es un signo que se refiere al Objeto que denota en virtud de estar realmente afectado por éste.

vi) Dicisigno: es un signo que, para su interpretante, es Signo de existencia actual.

vii) Legisigno: es una Ley que es un Signo, esta Ley es creación de la humanidad, no es un Objeto singular sino un tipo general que, por convención, será significante.

viii) Símbolo: es un Signo que induce a interpretar el símbolo como referido al Objeto.

ix) Argumento: es un Signo entendido como la mera representación de su Objeto por los caracteres propios de este.

El signo documental es símbolo en tanto que refiere a un objeto al que representa; índice porque deriva del objeto mismo (especialmente en recurso visuales); rhema porque posee una naturaleza cualitativa y conceptual; dicisigno por su naturaleza actual y relevante en un marco de conocimiento y argumento porque posee caracteres propios de los Objetos que representa y de los cuales quiere difundir un conocimiento

\subsection{La indización de los recursos de información}

La indización de los recursos de información en las bibliotecas se asume como una representación temática de las cosas por sí mismas a la vez que se considera un constructo que refleja cierta concepción del mundo (Davis, Shrobe \& Szolovitz, 1993). Rosa San Segundo dice que es "la descripción de contenido de los libros y documentos [...] de modo que los objetos representadores funcionan como sustitutos de los objetos representados" (San Segundo, 2003, p. 398). Mientras que Alan Gilchrist la observa como la sustitución de un elemento dentro de un modelo por la acción del vocabulario (Gilchrist, 2011).

La representación temática de los recursos de información posee tres funciones asociadas con principios generales de la disciplina bibliotecológica:

1. Función de estandarización: la representación documental busca ajustar las características de un Objeto dentro de un modelo establecido (estándares, normas, etc.), con la finalidad de que el producto del proceso (registro bibliográfico, registro documental, esquema de metadatos.) sea "un sustituto del correspondiente Objeto" (Lancaster y Pinto, 2001, p. 103).

2. Función de categorización: las representaciones construyen categorías que permiten acceder a todos los documentos incluidos dentro del sistema de organización de la información en relación a un valor establecido, esto es: autor, título, tema, colección, etc. (Sauperl, 2002).

3. Función información-conocimiento: se hacen representaciones para posibilitar el acceso a los recursos de información para una comunidad de usuarios. Se les ofrece información que, en contextos específicos, puede transformarse en conocimiento (Sauperl, 2002).

En la indización de los recursos de información, según el proceso establecido por Gil Leiva (2008), los elementos constitutivos son:

- Los recursos de información: que serán representados

- El representador: idealmente con formación profesional y experiencia en la actividad.

- El análisis documental temático: mediante el cual se determina el aboutness del recurso. 
- Los lenguajes de indización: libres o controlados que serán usados para concretar el aboutness del recurso en signos lingüísticos.

- El sistema para la gestión de los recursos de información: que puede ser manual o automatizado.

- El usuario: que intenta recuperar recursos desde un enfoque tópico.

El proceso de representación temática de los recursos de información contempla las siguientes etapas (Gil Leiva, 2008):

1. Contacto con el recurso: consiste en la revisión de áreas esenciales del documento (portada, índice, etc.), la lectura de páginas preliminares y complementarias e, incluso, la consulta de capítulos o partes de la obra que contribuirán para el establecimiento del aboutness.

2. Definición mental del aboutness: tan pronto como el recurso es conocido, se está en posibilidad de esbozar una idea mental al respecto (Hutchins, 1978). Ésta puede ser precisa o, en ocasiones, algo difusa en función del documento, por ejemplo, "el recurso trata sobre células procariontes" o "el recurso trata sobre diversos tipos de violencia contra las personas en situación vulnerable, como mujeres, niños en situación de calle, personas con preferencias sexuales diversas y recoge distintos tipos de violencia, como violencia psicológica, física, verbal, etc."

3. Asignación de temas: es el momento en que el representador concreta el contenido temático del recurso en una manifestación significativa. Para ello, puede emplear el lenguaje libre, extraer palabras o frases del recurso o seleccionar términos de algún vocabulario controlado (encabezamientos de materia, tesauros, taxonomías, etc.).

En el proceso de indización el signo está en lugar de su Objeto, pero "no en todos los aspectos, sino sólo con referencia a una suerte de idea, que a veces es llamada el fundamento del representamen" (Peirce,1974, p. 22). De manera que cada signo tiene un precepto que lo hace ser entendido como la expansión de su Objeto.

\subsection{Aspectos semióticos subyacentes} a la representación temática de los recursos de información

La indización de los recursos de información es formalmente un proceso de semiosis que integra todos los elementos constitutivos de la triada establecida por Peirce (Figura 3). Como se observa en la figura, la representación temática de los recursos de información contempla: un Objeto (recurso de información) al que el representador a partir de un Fundamento (proceso de escrutinio y análisis) le confiere un Signo (economía marxista) que lo representa en un sistema organizador de información. También incluye al Interpretante que es un nuevo signo o conjunto de signos motivados por el objeto en la mente de quien recibe el signo original.

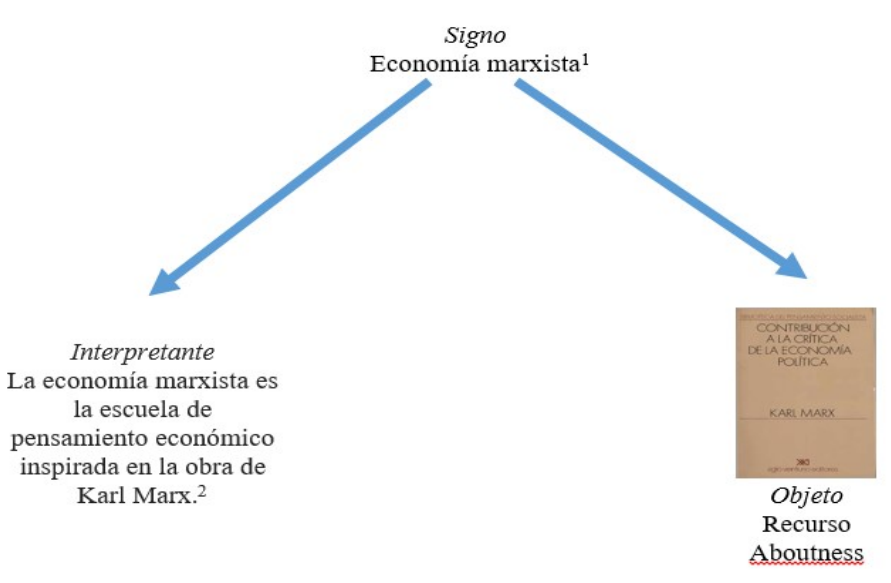

Figura 3. Modelo semiótico y representación temática de recursos

En la indización temática de las entidades bibliográficas se evidencian las tres categorías o niveles del modelo semiótico de Peirce: primeridad, segundidad y terceridad.

Primeridad: es el modo del ser de aquello que es tal como es, de manera positiva y sin referencia a ninguna otra cosa. En la primeridad, el indizador define el Objeto mediante una aproximación sensorial, sin referencia a ninguna cosa y examina sus partes esenciales: título, portada, índice, etc., para detectar su contenido temático.

Segundidad: es el modo de ser de aquello que es tal como es con respecto a una segunda cosa, pero con exclusión de toda tercera cosa. En la segundidad, el profesional de la información va más allá de lo que el recurso es, con respecto a una segunda cosa. Llegados a este punto es importante mencionar que las cosas son a partir de sí mismas y a partir de los que no son o en comparación con un segundo elemento. Por ejemplo, el tema de una obra titulada "Huracanes" puede ser "fenómenos naturales meteorológicos" porque conceptualmente no corresponde con la denominación conceptual de tal término. En este segundo nivel también son consideradas como segunda cosa la división de las disciplinas y la estructuración del conocimiento que los individuos y la sociedad han construido a la largo del tiempo.

Terceridad: es el modo de ser de aquello que es tal como es, al relacionar una segunda y tercera cosa entre sí. Por último, la terceridad refiere al 
modo de ser una entidad al relacionar una segundidad y terceridad, es decir, las relaciones a nivel tópico que la entidad informativa puede establecer.

\subsubsection{El Objeto}

El Objeto, bajo el modelo de Peirce, es algo sobre lo cual el Signo presupone un conocimiento. En la realidad puede ser cualquier cosa real o abstracta mientras que en la representación temática de los recursos "el tema de un documento es visto como el Objeto, ese "algo", en lo que el análisis temático se enfoca y trata de identificar" Hjørland (1997, p. 6). Como resultado, en el ejemplo presentado en la Figura 3 el Signo "Economía marxista" tiene un Objeto al que representa y su relación con el Objeto es imbuida, es decir, no se relaciona con el Objeto-Soporte sino con un Objeto-Temático imbuido en este (aboutness). Así, el Signo adecuado para la representación temática de la entidad bibliográfica no es "Libro" sino "Economía marxista".

\subsubsection{El signo}

En la relación del Signo-Objeto según Peirce, los signos se adhieren a tipologías diversas. En comparación, la caracterización del Signo bibliotecológico es amplio en algunas etapas y reducido en otras. Es amplio en la recepción del primer Signo, cuando el catalogador se enfrenta a recursos de información en los que hay elementos textuales, visuales, sonoros, etc. Pero, puede tornarse reducido cuando el profesional debe concretar el contenido temático de los recursos en expresiones lingüísticas.

Tal reducción se incrementa si en la indización se emplean vocabularios controlados (encabezamientos de materia, tesauros, taxonomías, ontologías terminológicas) cuya función es "contribuir para una indización eficiente" (Gil Leiva, 2008, p. 116). El empleo de vocabularios controlados para concretar las representaciones temáticas es útil en tanto que "posibilita la representación consistente de las materias por parte de los indizadores" (Lancaster, 1995, p. 22). Empero, su uso reduce considerablemente las posibilidades de signos lingüísticos que el indizador puede emplear para concretar el contenido temático.

Como señala Mai (2001), el principal problema en la representación temática de los documentos en aras de su recuperación estriba en la previsión del significado y el lenguaje, específicamente en cómo una declaración temática puede ser representada usando unas cuantas palabras o símbolos. Para los indizadores la vinculación entre Signo y Objeto es una tarea compleja debido a dos aspectos: la determinación respecto a ¿de qué trata el Objeto bibliográfico? y la selección del Signo adecuado. Cleveland y Cleveland (2013) establecen que una indización de calidad es el resultado de una serie de buenas decisiones del indizador quien decide qué tópicos realmente capturan el contenido temático del recurso.

Otro asunto complejo al que se enfrentan los indizadores en la vinculación Signo-Objeto es la falta de univocidad. De manera idónea a cada Objeto le debería corresponder un Signo, pero frecuentemente no es así, obsérvese el caso siguiente:

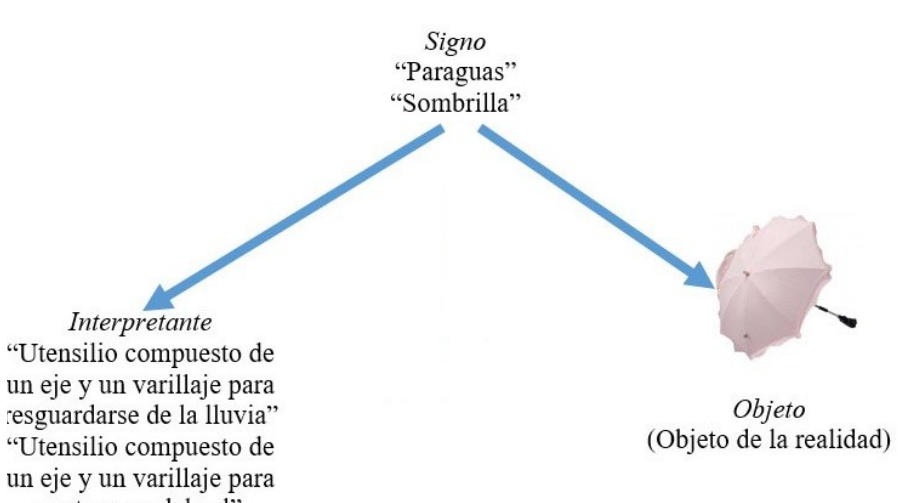

n eje y un varillaje para protegerse del sol"

Figura 4. Relación Objeto-Signo-Interpretante

Según la semiótica, un Objeto puede tener más de un Signo, a partir de la percepción del representador. Así, en la figura anterior (Figura 4), para una entidad que alguien observa se pueden presentar dos Signos (Paraguas y Sombrilla). Tal hecho se extiende a la bibliotecología donde persiste la falta de univocidad en torno a la representación temática de los recursos de información. Derivado de ello, "uno de los mayores problemas que plantea la consulta de bases de datos bibliográficos es, sin duda, el de la diversidad de puntos de acceso. Las distintas formas que el catalogador puede escoger a la hora de fijar éstos son tantas como agencias bibliográficas combinadas" (Agenjo, 1991, p. 241).

En el ámbito bibliotecológico, los signos que sustituyen temáticamente a las entidades bibliográficas forman índices a la vez que son elementos de búsqueda mediante los cuales un usuario recupera recursos para satisfacer sus necesidades de información. Forman parte de un proceso constituido por dos momentos cruciales: la indización y la búsqueda de los recursos de información.

En la indización, primero, un profesional de la información analiza el documento y construye una 
idea mental de este, por ejemplo: El origen de las especies de Carlos Darwin "trata sobre el principio de las especies a partir del proceso de adaptación y la selección natural". En consecuencia, los símbolos empleados para su representación pueden ser "Evolución (Biología)" y "Selección natural".

En la búsqueda de temática de recursos, posteriormente, el usuario tiene en su mente una noción o conceptualización que denominamos necesidad de información, por ejemplo, "para mi ensayo semestral necesito algunos materiales que traten sobre la teoría de un autor que el profesor mencionó que se llama Carlos Darwin que dijo que los seres vivos evolucionan". Pero, para que ésta sea planteada en un catálogo bibliotecario o sistema recuperador de información requiere su concreción en un Signo preciso. La concreción del Signo que el usuario empleará para buscar por temas es un asunto complejo (Suárez, 2011, p. 91):

[...] los usuarios tienen graves problemas en la formulación de búsqueda. Son poco hábiles trasladando conceptos a símbolos comprensibles para el sistema de recuperación de información. Para la mayoría es sencillo dar una descripción del tema que necesitan, por ejemplo: "mi tema es sobre regionalismos, pero no relacionados con la política, sino con la literatura" [...] Su problema es la traducción del concepto en palabras de búsqueda [...] Así, para el tema el usuario buscaría por "Regionalismos", "Tradición" y "Literatura".

El Signo se torna un elemento esencial para el éxito del proceso recuperador, pero en la bibliotecología se ha investigado muy poco sobre cómo un usuario define los signos con los que interroga un sistema y si tal Signo tiene correspondencia con lo que mentalmente estableció como su necesidad de información. De igual modo, tampoco se ha estudiado si el indizador considera la extensión del Signo hacia la búsqueda de los recursos. Mediante signos a los que denominamos descriptores, encabezamientos de materia, palabras clave, etc., asumimos, en la idoneidad, que existe un proceso de comunicación claro entre el representador y el receptor en términos del Interpretante, o aquello que el Signo determina, pero es necesario ir más allá en el estudio.

Aunque en documentación se persigue la normalización del Signo, el ideal se desvanece frente a la complejidad del proceso desde la parte conceptual y simbólica. Factores inherentes a la actividad en sus orígenes -como la configuración conceptual del Objeto y la dispersión terminológica imposible de concretarse en manifestaciones léxicas abarcadoras- se transforman en obstáculos para la normalización de los puntos de acceso. Ante la falta de univocidad representadora, resulta valioso el trabajo que las unidades de información emprenden para el control terminológico como son los catálogos de autoridades, los vínculos de referencias cruzadas y los anillos semánticos que minimizan el problema.

La indización de recursos de información es una actividad esencial para su recuperación temática en un sistema. Es definida frecuentemente como un proceso que conlleva una serie de pasos; sin embargo, sus fundamentos van más allá en tanto que el Signo forma parte del lenguaje humano y, como tal, es un elemento clave en la producción, representación, organización y comunicación del conocimiento.

\subsubsection{El interpretante}

El interpretante es "alguien o algo" a quien el signo se dirige y en quien crea una idea mediante otro signo o un signo más complejo. En gran medida, está asociado a un proceso cognitivo o de razonamiento. Tal interpretante puede ser de tres tipos: a) inmediato, cuando el Signo es aplicable a algo que el receptor conoce suficiente; b) dinámico, el Signo experimentado en cada acto de interpretación; y c) final: efecto que el signo produce sobre cualquier mente en circunstancias que permiten ejercer su efecto pleno.

En la triada Objeto-Signo-Interpretante, este último es un concepto discutido y hasta incomprendido (Sebeok, 1996); no obstante, si algo queda claro a partir de los textos de Peirce es que el Signo determina un Interpretante que a la vez puede ser bien un Signo equivalente u otro más desarrollado. Sebeok nos ofrece una excelente clarificación cuando a partir del Signo "Caballo" deduce tres interpretantes: "potro", "animal ungulado perisodáctilo cuadrúpedo" y "Equus ferus caballus". El Interpretante es lo que el Signo crea en la mente del receptor que, para determinarlo, a su vez, se vale de uno o varios signos. Volvemos a la premisa de que el Signo abarca toda noción de pensamiento asociado a lo abstracto o lo real y no hay pensamiento sin Signo.

En la representación temática de los recursos de información el Interpretante es altamente complejo y está presente de manera continua el proceso de semiosis cuando: (1) el creador de la obra percibe la realidad y sus signos y la recrea en una obra intelectual; (2) cuando el indizador analiza los documentos y los interpreta; (3) cuando profesional determina el contenido temático de la obra y crea representaciones mediante signos que engloban su contenido temático; (4) cuando el usuario del sistema consulta los índices o campos temáticos y los interpreta. En la bibliotecología quien recibe el 
signo puede ser un usuario humano, pero también es posible un interpretante artificial (computadora, buscador, etc.) que sólo posee un nivel simbólico para la recepción del signo.

\subsubsection{El representador}

El proceso de semiosis puede tener como representador un sujeto común que utiliza los signos para representar y comunicar cosas que observa, piensa o imagina o bien puede ser especializado, por ejemplo, un biólogo que analiza una población de especies de tortugas y les asigna signos en un código especializado.

En la indización de los recursos de información, como actividad profesionalizante, el representador ideal debe ser un especialista a quien su formación profesionalizante y su experiencia le permitan definir con precisión aquellos signos que serán atribuidos a los objetos bibliográficos. Su expertise es fundamental en la vinculación del Objeto-Signo más adecuada, puesto que existe una evidente relación entre la experiencia del catalogador y el nivel de exhaustividad, especificidad y consistencia de la indización (López, 2004).

\subsubsection{La semiosis}

Semiosis es cualquier actividad o proceso que involucra signos para la creación de un significado e implica una relación triádica entre un signo o representamen, un objeto y un interpretante. En la representación temática de los recursos de información estamos en una relación Signo-Objeto-Interpretante como sigue (Figura 5, en la página siguiente).

La indización de los recursos de información "puede ser expresada en términos de Peirce como una semiosis ilimitada: cada elemento del proceso de indización temática debe ser considerado como un signo y cada paso funciona como un acto de interpretación que une los signos en un proceso secuencial (Mai, 2001, p. 603). El proceso parte del documento como signo inicial, el indizador hace un acto de interpretación, y el producto de tal acto es un nuevo signo que el usuario interpreta en un sistema recuperador de información. La representación temática de los recursos de información posee vínculos muy cercanos con la recuperación temática de los mismos en tanto que el Signo es el elemento que une la necesidad de información del usuario con aquellos recursos que la satisfagan. Aunado a ello, la recuperación temática sólo obtiene resultados si el Signo expresado por el usuario registra coincidencia (matching) con algún Signo establecido por el indizador en el sistema.

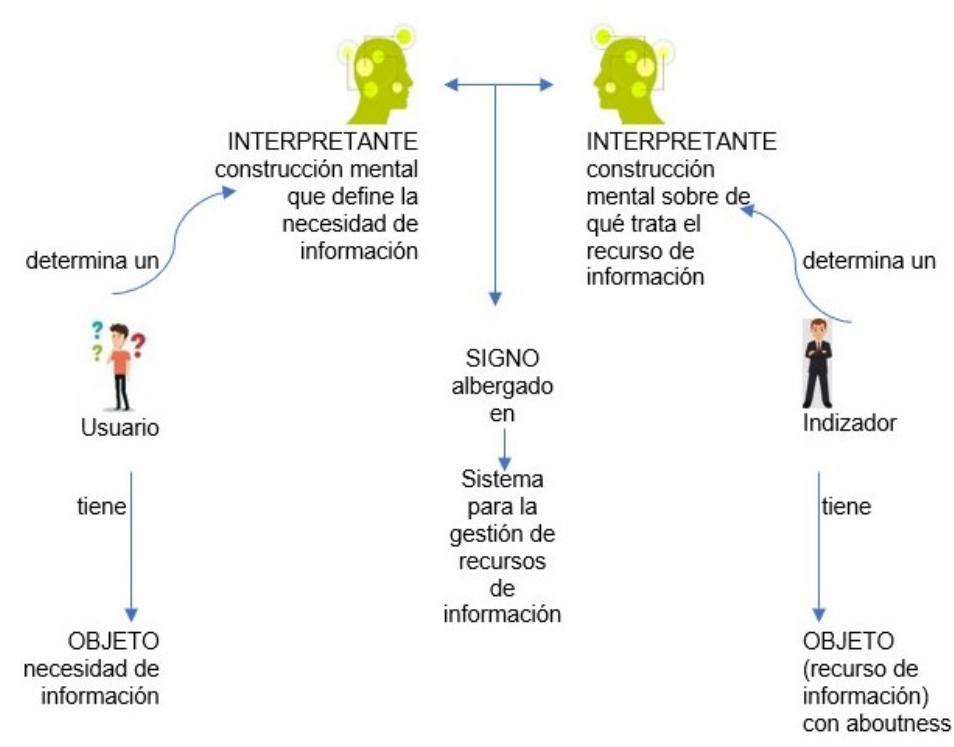

Figura 5. Semiótica y representación temática

La representación temática de los recursos de información es una semiosis ilimitada en la que cada elemento debe considerarse como asignado y cada paso funciona como un acto de interpretación que vincula los signos en un proceso secuencial (Mai, 2001). La actividad parte de un signo inicial: el documento. El indizador interpreta el primer signo y produce un segundo signo: el tema. Posteriormente, la semiosis continúa cuando el usuario se acerca a los campos temáticos o índices, observa los encabezamientos o descriptores asignados y en un acto de interpretación analiza las declaraciones temáticas de los recursos.

Un último aspecto de la semiosis que se extiende a la indización de los recursos de información es la dispersión representadora. Retomo a Mai (2001, p. 620), cuando establece que "el análisis de un documento involucra una clase de interpretación que es altamente dependiente del contexto cultural y social del indizador y del proceso de indización". Cleveland y Cleveland (2013) coinciden al respecto cuando señalan que el indizador aplica su esfuerzo intelectual para asignar términos o descriptores a un recurso, en gran medida su trabajo se fundamenta en estándares y procesos para realizar su labor; no obstante, persiste una interpretación subjetiva de los conceptos implicados en el documento.

\section{Conclusiones}

De ser la ciencia del Signo como elemento epistemológico constructor, la semiótica se ha convertido en la ciencia de las significaciones (Vidales Gonzáles, 2010). En este sentido, lo que tenemos 
hoy es una teoría semiótica que se expande a diversas disciplinas como la psicología, la literatura, la comunicación, la mercadotecnia, etc.

La semiótica también alcanza a la bibliotecología que es un campo de conocimiento representador y comunicador, inserto en las ciencias y los estudios de la información. Derivado de los resultados obtenidos en esta investigación, es posible decir que hay un alto grado de aspectos semióticos subyacentes en la representación temática de los recursos de información. Los elementos Objeto-Signo-Interpretante se mantienen prácticamente intactos; no obstante, hay ciertos elementos caracterizadores del proceso representador: el tipo de Objeto con el que se está trabajando, las características del representador y su recepción por parte de "alguien" a quien está dirigido, que conceptualizamos como un usuario con una necesidad informativa.

El modelo semiótico de Peirce puede ser muy relevante para los bibliotecólogos en tanto que permite observar nuevos ángulos de la representación temática de recursos. Por lo tanto, según Almeida, Lopes Fujita \& Dos Reis (2013, p. 226).:

[...] necesitamos otra forma de entender el proceso de indexación, una perspectiva que agregue la producción de signos como una actividad que guía el proceso de indexación [...] Esto implica que la indexación no es solo es una acción representativa cuyo objetivo es establecer palabras relacionadas con conceptos con fines de control de vocabulario y útiles para la recuperación de información y los sistemas de organización. Nuestro argumento es que la indexación temática es un proceso de representación y, por lo tanto, requiere teorías de varias disciplinas, a saber: lingüística, semiología, filosofía del lenguaje, lógica y semiótica.

El interés en el Signo desde perspectiva bibliotecológica ha sido evidenciado por varios autores. Svenonius $(2000$, p. 6$)$ dice que "el acto de organizar información puede ser visto como un tipo particular de uso del lenguaje". Hørland (1997, p. 17) coincide al respecto cuando afirma que "los documentos contienen textos, en un sentido generalizado, (incluyendo, por ejemplo, fotografías) escritos por otras personas. El estudio de éstos debe construirse sobre algún tipo de teoría de la comunicación y los signos (semiótica)".

En bibliotecología el Signo es relevante porque está asociado a dos funciones: representación y comunicación. A partir de la función representadora, se concreta en registros bibliográficos que sustituyen a los recursos de información en un sistema. A partir de la función comunicativa es un puente que une las necesidades de información de los usuarios con los recursos idóneos que las satisfagan.
Actualmente, las ciencias se encuentran en un estado de confluencia de muchas disciplinas y la bibliotecología no es la excepción, en vista de que puede beneficiarse de las ciencias computacionales, la lingüística, la filosofía, la psicología y otras áreas que deriven en el enriquecimiento de las actividades que se realizan en las bibliotecas y la formación de profesionales capaces de superar los retos asociados a la organización de la información hoy.

\section{Notas}

(1) La representación temática del recurso "Economía marxista" fue tomada del catálogo bibliotecario LIBRUNAM que alberga las colecciones de libros de la Universidad Nacional Autónoma de México y está a cargo de la Dirección General de Bibliotecas de la Institución.

(2) La interpretación del signo "Economía marxista" fue extraída de Wikipedia.

\section{Referencias}

Almeida, Carlos Cándido (2012). The methodical influence of Peirce's pragmatism on knowledge organization. II Knowledge Organization. 39:3 (Diciembre 2012) 204-215.

Almeida, Carlos Cándido, Lopes Fujita, Mariângela Spotti. \& Dos Reis, Daniela Majorie (2013). Peircean semiotics and subject indexing of speculative grammar and pure logic. // Knowledge Organization. 40:4 (Enero 2013) 225-241.

Agenjo Bullón, Xavier (1991). La catalogación cooperativa en el entorno de la interconexión de sistemas abiertos. // Boletín de la ANABAD. 41:3-4 (Enero 1991) 241-248.

Cleveland, Donald; Cleveland, Ana (2013). Introduction to indexing and abstracting. 4a.ed. Santa Barbara, California: Libraries Unlimited, 2013.

Conesa, Francisco; Nubiola, Jaime (2002). Filosofía del lenguaje. $2^{\mathrm{a}}$ ed. Barcelona: Herder, 2002.

Davis, Randall; Shrobe, Howard; Szolovits, Peter (1993). What is a knowledge representation? // Artificial Inteligence Magazine. 14:1 (Marzo 1993) 17-33.

Feather, John; Sturges, Paul (2003). International encyclopedia of information and library science. Londres: Routledge, 2003.

Ferrater Mora, Jose (1999). Diccionario de filosofía. BarceIona: Ariel, 1999

Gil-Leiva, Isidoro (2008). Manual de indización: teoría y práctica. Gijón. TREA, 2008.

Gilchrist, Alan (2011). A note on the theory and practice of knowledge organization and knowledge representation. // Scire: representación y organización del conocimiento. 17:1 (Enero 2011) 23-28.

Hjørland, Birger (1997). Information seeking and subject representation. Westport, Connecticut: Greenwood Press, 1997.

Hutchins, William John (1978). The concept of 'aboutness' in subject indexing. // Aslib Proceedings. 30:5 (Mayo 1978) 172-181.

Izquierdo Arroyo, José María (1993). De la semiótica del discurso a la semiótica documental. // Moreiro Gonzalez, José Antonio (ed.). Aplicaciones de las ciencias del texto al re-sumen documental. Madrid. Universidad Carlos III, 1993.199-216.

Lancaster, Frederick (1995). El control de vocabulario en la recuperación de información. Valencia: Universidad de Va-lencia, 1995. 
Lancaster, Frederick; Pinto, María (coord.) (2001). Procesamiento de la información científica. Madrid: Arco Libros, 2001.

Lopez Yepes, José (ed.) (2004). Diccionario enciclopédico de ciencias de la documentación. Madrid: Síntesis, 2004.

Martínez de Souza, José (2004). Diccionario de bibliología y ciencias afines. 3a . ed. Gijón: TREA, 2004.

Mai, Jens-Erik (2000). The subject indexing process: an inves-tigation of problems in knowledge representation. Tesis doc-toral. Universidad de Texas en Austin. https://pdfs.semanticscholar.org/5d25/032bac9c41ac2c1 653be2c35ea2d9a8ea0ce.pdf (2020-06-28).

Mai, Jens-Erik (2001). Semiotic and indexing: an analysis of the subject indexing process. // Journal of Documentation. 57:5 (Septiembre, 2001) 591-622.

Niño Rojas, Víctor Manuel (2013). Semiótica y lingüística. Bogotá: ECOE, 2013.

Ogden, Charles Kay; Richard, Ivor Armstrong (1953). El signifi-cado del significado: una investigación acerca de la influencia del lenguaje sobre el pensamiento y de la ciencia simbólica. Barcelona: Paidós, 1984.

Peirce, Charles Sanders (1974). La ciencia de la semiótica. Buenos Aires: Nueva visión, 1974.

San Segundo Manuel, Rosa (2003). Nueva concepción de la representación del conocimiento. // Travieso Rodríguez, Críspulo; Frías Moya, José Antonio (coords.). Tendencias de investigación en organización del conocimiento. Salamanca: Universidad de Salamanca, 2003. 395-402.

Sauperl, Alenka (2002). Subject determination during the cataloging process. Londres: Scarecrow, 2002.
Saussure, Ferdinand (1961). Curso de lingüística general. Buenos Aires: Losada, 1961. http://fba.unlp.edu.ar/lenguajemm/?wpfb_dl=59 (2020-01-22).

Sebeok, Thomas (1996). Signos: una introducción a la semióti-ca. Barcelona: Paidós, 1996.

Serrano, Sebastiá (1981). La semiótica: una introducción a la teoría de los signos. Barcelona: Montesinos,1981.

Suárez Sánchez, Adriana (2011). El lenguaje de indización y el lenguaje de la búsqueda por temas: análisis de su encuentro en la recuperación temática. Ciudad de México, México: El Colegio de México, 2011.

Svenonius, Elaine (2000). The intellectual foundations of infor-mation organization. Cambrige, Massachussets: Instituto de Tecnología de Massachusets, 2000.

Svenonius, Elainel (2004). The epistemological foundations of knowledge representations. // Library Trends. 52:3 (Diciem-bre 2004) 571-587.

Thiebaut, Carlos (2007). Conceptos fundamentales de filosofía. $2^{\mathrm{a}}$ ed. Madrid: Alianza, 2007.

Toledo Almada, Aletse; Sequera Meza, José Antonio (2014). La producción del sentido: semiosis social. // Razon y palabra. 88 (Enero 2014) 1-20.

Vidales Gonzáles, Carlos (2010). Semiótica y teoría de la comunicación. Nuevo León, México: CECYTE, 2010.

Enviado: 2020-02-10. Segunda versión: 2020-11-19. Aceptado: 2020-11-19. 
